\title{
Conversion of Nonlinear Quadcopter Mathematical Model to Linear Simplified Model
}

\author{
M Rehan Khan ${ }^{1}$, Sidra Malik ${ }^{2}$ \\ ${ }^{1}$ Institue of Avionics \& Aeronautics (IAA), Air University, Islamabad \\ ${ }^{2}$ Preston University, Islamabad
}

\begin{abstract}
Quadcopter control is a fundamentally difficult and interesting problem. With six degrees of freedom (three translational and three rotational) and only four independent inputs (rotor speeds), quadcopters are severely under actuated. In order to achieve six degrees of freedom, rotational and translational motion are coupled. The resulting dynamics are highly nonlinear, especially after accounting for the complicated aerodynamic effects. Finally, unlike ground vehicles, helicopters have very little friction to prevent their motion, so they must provide their own damping in order to stop moving and remain stable. Together, these factors create a very interesting control problem. We will present a very simplified model of quadcopter and removes nonlinearity from system modeling by using tayler expansions and trigonometric identies.
\end{abstract}

Keywords: Translational, Rotational, Quadcopter, nonlinear, dynamic

\section{Introduction}

A quad copter, also referred to as drone, is a type of aerial vehicle that uses four motors to hover and fly. It can be controlled remotely by an operator, or fly autonomously with a flight plan. Quad copters have many different applications; search and rescue missions, aerial photography and recreational flying. They offer one significant advantage over conventional helicopters since they lack all the complex and expensive mechanical linkage used in helicopters, which reduces the price. Quad copters lack a natural state of equilibrium, and therefore require a control system to stabilize it.

In order to carry out mathematical analyses and propose control laws, a mathematical model of the vehicle was required. Since the vehicle moves in $6 \mathrm{DoF}$ (six-degrees of freedom), a suitable coordinate system handling these freedoms was needed.

Quad copter was conceived for the purpose of nondestructive evaluation of hard to reach structures. By carrying equipment on a highly mobile platform to areas that a human being cannot easily reach without the aid of additional equipment such as ladders, ropes and harnesses, and lifts, Quad copter will be able to greatly reduce the amount of time it takes to inspect a bridge or building. By diminishing time required and streamlining the inspection process, Quad copter could allow inspectors not only to conduct more thorough inspections, but also to conduct more inspections in a shorter amount of time, which could in turn help to prevent disasters such as the Interstate 35 bridge collapse. The end result would be that the safety and peace of mind that people expect when traveling would be nearly complete

\section{System Model}

In this section, the quad rotor system's multiple inputs and outputs will be identified and the equations of motion governing the dynamics of the system will be derived. The first step is to identify the plant and the inputs and outputs of the plant itself and then to establish the framework to derive the equations of motion using Newton's Laws.

A. Plant Identification

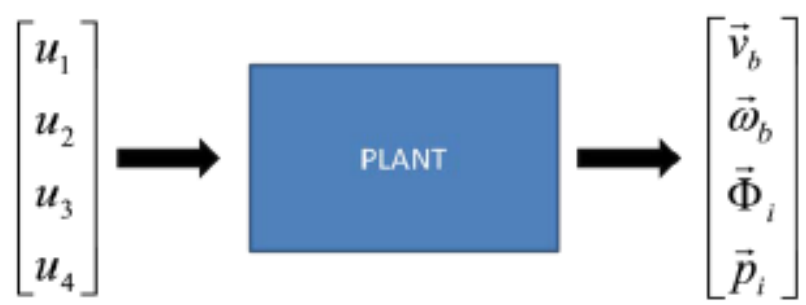

Figure 1: Plant Inputs and Outputs

Figure 1 shows the inputs and outputs of the plant. The inputs are angular velocities in radians per second and the output is a $12 \times 1$ vector which is discussed below. The angular velocity is converted through an airfoil blade. The Dynamics of the airfoil blade is included in the dynamics of the plant. In order to control the angular velocity of the blades, we must control the voltage applied to the motors in the quad rotor. Below shows how the motors can be controlled through pulse-width modulation (PWM) the axel of the motor is geared with a five to one reduction gear. The result of varying duty cycles of the motor drive stage results in varying angular velocities.

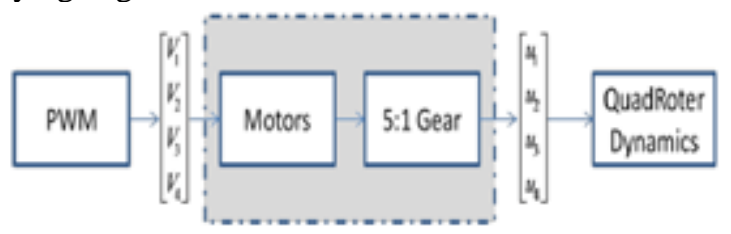

Figure 2: Motor and gear control

\section{B. Reference Frame}

The quad rotor system operates in two coordinate frames: inertial and body. The inertial frame (also referred to as the earth frame) is the coordinate axis where Newton's Laws apply. To complicate matters, the countering forces to achieve hover are applied to the body frame which is fixed to 


\section{International Journal of Science and Research (IJSR) \\ ISSN (Online): 2319-7064}

Index Copernicus Value (2013): 6.14 | Impact Factor (2015): 6.391

the quad rotor itself and is allowed to rotate and translate. This dual-frame coordinate system is shown below along with a free body diagram of the quad rotor system.

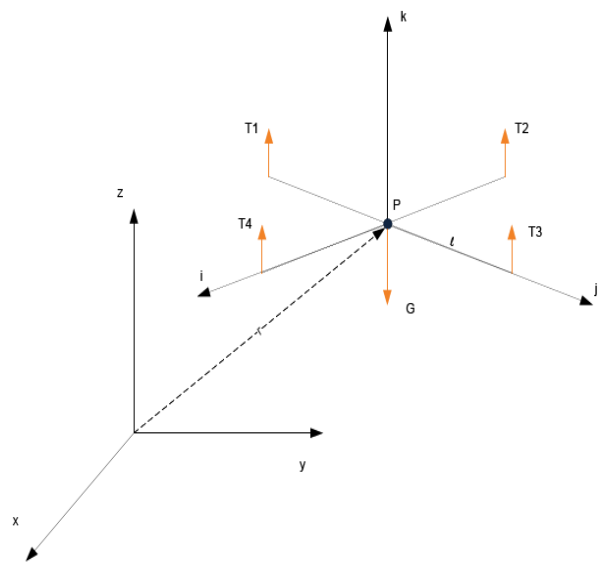

Figure 3: Reference Frame

Now that the coordinate reference frame is identified we can begin to represent the system mathematically. The Mechanism through which the quad rotor can be controlled is thrust provided by airfoil blades attached to four independently controllable motors attached at a fixed distance (l) from the center of the quad rotor $(\mathrm{P})$. By varying the relative magnitudes of the thrusts, we can control the attitude (yaw, pitch, roll) and position (X,Y,Z) of the system in inertial coordinates. As mentioned previously, the thrust forces are applied in the body frame; therefore, transformations must be made.

\section{Mathematical Model}

Now we define the states of the system that include a mixture of body and inertial components comprised of translational and rotational positions and velocities. We define the following

$\sum F=m \ddot{x}$

$\sum M=I \ddot{\theta}$

Above equations are for inertial frame

$U=$ Tangential Velocity in body frame $\hat{\imath}$

$V=$ Tangential Velocity in body frame $\hat{\jmath}$

$W=$ Tangential Velocity in body frame $\hat{k}$

$P=$ Rotational Velocity in body frame $\hat{\imath}$

$Q=$ Rotational Velocity in body frame $\hat{\jmath}$

$R=$ Rotational Velocity in body frame $\hat{k}$

$\bar{v}^{b}=\left[\begin{array}{l}u \\ v \\ w\end{array}\right]$

$\bar{w}^{b}=\left[\begin{array}{l}p \\ q \\ r\end{array}\right]$

$\Phi=\left[\begin{array}{l}\varphi \\ \theta \\ \psi\end{array}\right]$

$\bar{r}=\left[\begin{array}{l}p_{i} \\ p_{j} \\ p_{k}\end{array}\right]$

Combining the four vectors defined above yields the state vector which is used in the derivation of the quad rotor dynamics shown in the appendix

\section{State Vector}

In the inertial frame, the acceleration of the quadcopter is due to thrust, gravity, and linear friction. We can obtain the thrust vector in the inertial frame by using our rotation matrix $X$ to map the thrust vector from the body frame to the inertial frame. Thus, the linear motion can be summarized as $X=\left[\begin{array}{c}\bar{v}^{b} \\ \bar{w}^{b} \\ \Phi \\ \bar{r}\end{array}\right]$

$\dot{X}=\frac{d}{d t} X$

By using newton's law

$\sum F=\left.m \frac{d}{d t}\right|_{i} \bar{v}^{b}$

$\left.\frac{d}{d t}\right|_{i} \bar{v}^{b}=\left.\frac{d}{d t}\right|_{b} \bar{v}^{b}+\left(\bar{w}^{b}+\bar{v}^{b}\right)$

So

$\sum F^{i}=\left.m \frac{d}{d t}\right|_{i} \bar{v}^{b}=\left.\frac{d}{d t}\right|_{b} \bar{v}^{b}+\left(\bar{w}^{b}+\bar{v}^{b}\right)$

$\left.\frac{d}{d t}\right|_{b} \bar{v}^{b}=\frac{1}{m} \sum F^{b}-\left(\bar{w}^{b}+\bar{v}^{b}\right)$

As

$\sum F=F_{G}+F_{T}$

$F_{G}{ }^{i}=\left[\begin{array}{c}0 \\ 0 \\ m g\end{array}\right]$

$F_{g}{ }^{b}=m g\left[\begin{array}{c}-\sin \theta \\ \cos \theta \sin \varphi \\ \cos \theta \cos \varphi\end{array}\right]$

$F_{T}{ }^{i}=\sum_{j=1}^{4} T_{j}$

$T_{j}=\check{b} \Omega_{j}^{2}$

$\Omega_{j}=$ angular velocity of blade

$F_{T}{ }^{b}=\left[\begin{array}{c}0 \\ 0 \\ -T\end{array}\right]$

$\frac{d}{d t}\left[\begin{array}{c}u \\ v \\ W\end{array}\right]^{b}=\frac{1}{m}(\underbrace{m g\left[\begin{array}{c}-\sin \theta \\ \cos \theta \sin \varphi \\ \cos \theta \cos \varphi\end{array}\right]^{b}}_{\text {Gravity }}+\underbrace{\left[\begin{array}{c}0 \\ 0 \\ -T\end{array}\right]^{b}}_{\text {Thrust }})-\left[\begin{array}{c}p \\ q \\ r\end{array}\right]^{b} X\left[\begin{array}{c}u \\ v \\ w\end{array}\right]$

So

$\frac{d}{d t}\left[\begin{array}{l}u \\ v \\ w\end{array}\right]=\frac{1}{m}\left(m g\left[\begin{array}{c}-\sin \theta \\ \cos \theta \sin \varphi \\ \cos \theta \cos \varphi\end{array}\right]+\left[\begin{array}{c}0 \\ 0 \\ -T\end{array}\right]^{b}\right)-\left[\begin{array}{l}p \\ q \\ r\end{array}\right] X\left[\begin{array}{l}u \\ v \\ w\end{array}\right]$

$=g\left[\begin{array}{c}-\sin \theta \\ \cos \theta \sin \varphi \\ \cos \theta \cos \varphi\end{array}\right]+\frac{1}{m}\left[\begin{array}{c}0 \\ 0 \\ -T\end{array}\right]-\left[\begin{array}{c}q w-r v \\ r u-p w \\ p v-q u\end{array}\right]$

$\frac{d}{d t}\left[\begin{array}{c}u \\ v \\ w\end{array}\right]=\left[\begin{array}{c}-g \sin \theta-q w+r v \\ g \cos \theta \sin \varphi-r u+p w \\ g \cos \theta \cos \varphi+\frac{1}{m T}-p v+q u\end{array}\right]$

Then resulted equations are

$$
\begin{gathered}
\dot{u}=r v-q w-g \sin \theta \\
\dot{v}=p w-r u+g \cos \theta \sin \varphi \\
\dot{w}=q u-p v+g \cos \theta \cos \varphi+\frac{b}{m}\left(w_{1}{ }^{2}+w_{2}{ }^{2}+w_{3}{ }^{2}+\right. \\
w 42
\end{gathered}
$$

The final nonlinear model of the quad copter is compactly presented here. 


\section{International Journal of Science and Research (IJSR) \\ ISSN (Online): 2319-7064}

Index Copernicus Value (2013): 6.14 | Impact Factor (2015): 6.391

\section{Linearized Model}

In order to apply linear control theory, a linear system is needed. Almost all systems are in some sense nonlinear. When approximating a nonlinear system by a linear one, it is important to determine if the approximation is good and can be motivated. By assuming that the quadcopter is kept within some equilibrium i.e., hovering, a linearization of the nonlinear system can be motivated. Furthermore, linear systems are often easier to control and to analyze and many popular control methods are only applicable on linear systems. Therefore, a linearization of the nonlinear system in this section is made. The linearized system can be derived by doing a first order T.E (Taylor expansion) about some equilibrium. For an arbitrary nonlinear system

$$
\begin{array}{ll}
u=u_{0}+\Delta u, & u_{0}=0 \\
v=v_{0}+\Delta v, & v_{0}=0 \\
w=w_{0}+\Delta w, & w_{0}=0 \\
p=p_{0}+\Delta p, & p_{0}=0 \\
q=q_{0}+\Delta q, & q_{0}=0 \\
r=r_{0}+\Delta r, & r_{0}=0 \\
\theta=\theta_{0}+\Delta \theta, & \theta_{0}=0 \\
\varphi=\varphi_{0}+\Delta \varphi, & \varphi_{0}=0 \\
\psi=\psi_{0}+\Delta \psi, & \psi_{0}=0
\end{array}
$$$$
\text { Trigonometric Identity }
$$$$
\operatorname{Cos}(\alpha+\beta)=\operatorname{Cos} \alpha \cos \beta-\operatorname{Sin} \alpha \sin \beta
$$$$
\operatorname{Sin}(\alpha+\beta)=\operatorname{Sin} \alpha \cos \beta+\operatorname{Cos} \alpha \sin \beta
$$$$
\text { From initial conditions and trigonometric identities }
$$$$
\operatorname{Sin}\left(x_{0}+\Delta x\right)=\operatorname{Sin} \Delta x=\Delta x
$$$$
\operatorname{Cos}\left(x_{0}+\Delta x\right)=\operatorname{Cos} \Delta x=1
$$$$
\text { So }
$$$$
\dot{u}=\left(r_{0}+\Delta r\right)\left(v_{0}+\Delta v\right)-\left(q_{0}+\Delta q\right)\left(w_{0}+\Delta w\right)-g \sin \left(\theta_{0}+\right.
$$$$
\Delta \theta)
$$$$
=\left(r_{0} v_{0}+r_{0} \Delta v+\Delta r v_{0}+\Delta v \Delta r\right)-\left(q_{0} w_{0}+q_{0} \Delta w+\right.
$$$$
\left.\Delta q w_{0}+\Delta q \Delta w\right)
$$$$
-g\left(\sin \theta_{0} \cos \Delta \theta+\operatorname{Sin} \Delta \theta \cos \theta_{0}\right)
$$$$
=\underbrace{\Delta r \Delta v}_{\text {very small }}-\underbrace{\Delta q \Delta w}_{\text {very small }}-g \sin \Delta \theta
$$$$
\text { sowe so we }
$$$$
\text { So }
$$$$
\text { neglect it neglect it }
$$

$\dot{u}=-g \sin \Delta \theta$

$$
\dot{u}=-g \Delta \theta
$$

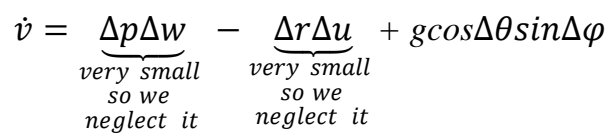

So

$$
\begin{gathered}
\dot{v}=g \Delta \varphi \\
\dot{w}=g \cos \Delta \theta \cos \Delta \varphi+\frac{b}{m}\left(w_{1}{ }^{2}+w_{2}{ }^{2}+w_{3}{ }^{2}+w_{4}{ }^{2}\right) \\
\dot{w}=2 w \Delta w
\end{gathered}
$$

\section{Conclusion}

The modeling of the quadcopter is crucial and has to be handled with care. The use of Euler angles and quaternions is quite common when expressing rotations in $6 \mathrm{DoF}$.. The modeled body forces and torques are determined to be among the most significant ones. Other prominent effects which are of interest, are aerodynamics. Extensive modeling regarding aerodynamics can be made. In this paper only quadcopter dynamics is modeled. In order to analyze and model other aerodynamic effects, experiments measuring properties of the vehicle have to be setup and performed. These experiments demand much care and preparation and would probably need an entire thesis project worth of time. Although, by having more knowledge of the vehicle's aerodynamics, better control algorithms could be derived. At the end of the modeling, the derived nonlinear model is linearized about hovering. When applying a first order T.E, all nonlinearities disappear and are not compensated for in a linear control. A solution to this would be to try to control the full nonlinear model. But since it is quite complex, it would not be as straight forward as the application of a linear controller. To simplify control, the nonlinear model can be approximated by a simpler nonlinear model. These nonlinear functions could be approximated by the small angle assumption which would simplify control, note that the resulting expression would still be nonlinear. Furthermore, it is also important to determine which nonlinearities should be compensated for. It might be desirable to leave some nonlinearities uncompensated.

\section{References}

[1] Torkel Glad and Lennart Ljung, ReglerteknikGrundla"ggande teori (Swedish), Studentlitteratur AB,Lund, 1981,2006, pp. 17-21, 173-176,184- 185.

[2] Henrik Ohlsson and Hrvoje Corluka. Modellering och reglering av en oktakopter. Department of Automatic Control, Lund University, Lund, Sweden. 2014.

[3] Kristoffer Bergman and Jonatan Ekström. Modeling, Estimation and Attitude Control of an Octorotor Using PID and L1 Adaptive Control Techniques. Department of Electrical Engineering, Linköpings universitet, Linköping, Sweden. 2014.

[4] J. G. Leishman, Principles of Helicopter Aerodynamics, Cambridge University Press, 2006, s. 183.

[5] F. Sørensen, Autonomous Control of a Miniature Quadrotor Following Fast Trajectories, 2010, s. 12.

[6] De Lellis, Marcelo, 2011. Modeling, Identification and Control of aQuadrotor Aircraft. Czech Technical University in Prague.

[7] Skogestad, S. \& Postlethwaite, I., 1996. Multivariable Feedback Control: Analysis and Design 1st ed., Wiley

[8] Castillo, P., Lozano, R. \& Dzul, A., 2005. Stabilization of a mini rotorcraft with four rotors. Control Systems, IEEE, 25(6), p.45- 55. Available at: [Accessed May 25, 2011].

\section{Author Profile}

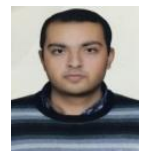

Mr. M.Rehan Khan M.S., is currently working as a Lab Engineer in the Department of Mechanical and Aerospace Engineering at Air University ,Islamabad ,Pakistan. He has about three years of teaching experience. His areas of specializations are Computational Fluid dynamics and thermal engineering.

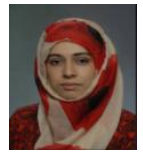

Ms.Sidra Malik MS., is currently working as a Lecturer in the Preston University, Islamabad, Pakistan. She has about two years of teaching experience. Her areas of specializations are Mathematical Modeling and Computational Fluid dynamics.

\section{Volume 5 Issue 6, June 2016 www.ijsr.net}

